\title{
Spectroscopic investigation of an intrinsic room temperature ferromagnetism in Co doped $\mathrm{ZnO}$ nanoparticles
}

\author{
Srinatha $\mathrm{N}^{\mathrm{a}}$, Basavaraj Angadi ${ }^{\mathrm{a}, *}$, K.G.M. Nair ${ }^{\mathrm{b}}$, Nishad G. Deshpande ${ }^{\mathrm{c}}$, \\ Y.C. Shao ${ }^{c}$, Way-Faung Pong ${ }^{c}$ \\ a Department of Physics, JB Campus, Bangalore University, Bangalore 560056, India \\ b UGC-DAE-CSR, Kalpakkam Node, Kalpakkam, Kokilamedu 603 102, India

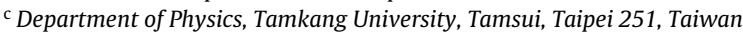

\section{A R T I C L E I N F O}

\section{Article history:}

Received 4 June 2014

Received in revised form 14 July 2014

Accepted 15 July 2014

Available online 23 July 2014

\section{Keywords:}

Dilute magnetic semiconductor

$\mathrm{ZnO}$

L-Valine

SQUID

NEXAFS

XMCD

\begin{abstract}
A B S T R A C T
Pure and Co substituted $\mathrm{ZnO}$ nano crystalline particles were prepared by solution combustion technique using L-Valine as a fuel. As synthesized powder samples were characterized by X-ray diffractometer and SQUID magnetometer to confirm the formation of single phase wurtzite structure and to study the bulk magnetic response of the sample, respectively. Magnetic studies show that Co doped ZnO nanoparticles exhibit ferromagnetism (FM) at room temperature (RT). Furthermore, the electronic structure and element specific magnetic properties were investigated by near-edge X-ray absorption fine structure (NEXAFS) and X-ray magnetic circular dichroism (XMCD) measurements, respectively. The effect of Co substitution on the spectral features of $\mathrm{Co}-\mathrm{ZnO}$ at $\mathrm{O} \mathrm{K}$-edge, $\mathrm{Co}_{3,2}$ edge, $\mathrm{Zn} L_{3,2}$ edge have been investigated. The spectral features of NEXAFS at $C_{0} L_{3,2}$ edge is entirely different from the spectral features of metallic clusters and other impurity phases, which rules out the presence of impurity phases. The valence state of ' $C o$ ' ion is found to be in +2 state. The FM nature of the sample was confirmed through XMCD spectra, which is due to the incorporation of divalent ' $\mathrm{Co}$ ' ions. Hence the presented results confirm the substitution of 'Co' ions at ' $\mathrm{Zn}$ ' site in the host lattice, which is responsible for the RTFM.
\end{abstract}

(c) 2014 Elsevier B.V. All rights reserved.

\section{Introduction}

Dilute magnetic semiconductors (DMSs) are the potential candidates for spintronic applications such as spin-polarized information storage and processing devices [1-3]. In order to realize the spintronic devices, the DMS's must exhibit ferromagnetism (FM) at room temperature (RT). Dietl et al. [4] and Sato et al. [5] predicted that, the transition metal ions doped $\mathrm{ZnO}$ are the promising candidates for spintronics applications with intrinsic DMS properties at RT. In this context transition metal doped ZnO DMS's are being extensively studied in the form of both nanoparticles [6-10] and thin films [11-17] by various research groups. Co doped ZnO based DMS materials are of great interest, due to the tunability of FM above RT, high solubility limit and large magnetic moment per 'Co' ion. But achieving single-phase, RTFM DMS is relatively difficult due to the formation of ' $\mathrm{Co}$ ' metallic clusters or other impurity oxide phases. However, theoretical and experimental controversies on

\footnotetext{
* Corresponding author.

E-mail addresses: brangadi@gmail.com, brangadi@bub.ernet.in (B. Angadi).
}

the issue of whether magnetism is intrinsic (carrier-induced) or extrinsic (secondary phase formation) are still prevailing. Theoretical calculations by Sako and Katayama-Yoshida [5], have predicted that intrinsic FM of Co doped $\mathrm{ZnO}$ can be achieved by electrondoping, when magnetic dopants substituted at ' $\mathrm{Zn}$ ' cation sites. There are few reports with experimental results on Co doped $\mathrm{ZnO}$ with DMS properties [6-17]. But the possibilities of extrinsic FM have not been ruled out, in fact it has been observed in Co doped $\mathrm{ZnO}$ [18-21], due to the formation of metallic Co impurity, $\mathrm{Co}_{3} \mathrm{O}_{4}$ phases and oxygen vacancies. Hence the origin of FM in Co doped $\mathrm{ZnO}$ is still under debate and not understood well yet. The near-edge X-ray absorption fine structure (NEXAFS) is an effective experimental tool and is an element specific technique, which can give information about the valance state, local environment, and hybridization of the specific cation (Co) in the material. On the other hand, X-ray magnetic circular dichroism (XMCD) can be used to probe information regarding the contribution of specific cation in any material toward the total magnetism of the system. It is also used to determine the spin and orbital contributions to the magnetism using sum rules $[22,23]$. In this work, we synthesized Co doped $\mathrm{ZnO}$ nanoparticles by solution combustion technique (SCT) using L-Valine as a fuel and 


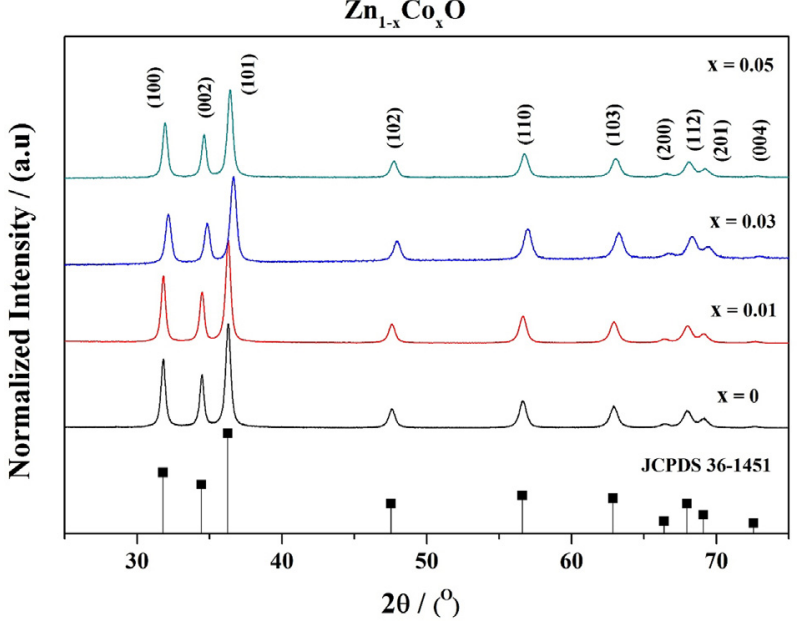

Fig. 1. The XRD pattern of as synthesized $\mathrm{Zn}_{1-x} \mathrm{Co}_{x} \mathrm{O}(x=0,0.01,0.03$ and 0.05$)$.

we report the intrinsic RTFM through NEXAFS and XMCD studies of the Co doped $\mathrm{ZnO}$ nanoparticles along with bulk superconducting quantum interference devices (SQUID) magnetic measurements.

\section{Experimental}

Pure and Co substituted ZnO nanoparticles were prepared by the SCT using Zinc Nitrate Hexa-hydrate as an oxidizer and L-Valine as a fuel and cobaltous nitrate hexa-hydrate as dopant. Stoichiometric amounts of oxidizer $(\mathrm{O})$ and fuel $(\mathrm{F})$ were taken based on the condition that the valance of $\mathrm{O} / \mathrm{F}$ to be unity, using total oxidizing and reducing valences of the oxidizer and the fuel. These stoichiometric amounts of starting materials dissolved in the double distilled water and stirred completely to get transparent solution. The transparent solution was dried on hot plate/muffle furnace at $100^{\circ} \mathrm{C}$ to remove water content in the solution. So obtained sticky solution (water free) was then placed in the pre-heated muffle furnace at $40{ }^{\circ} \mathrm{C}$ for combustion process. Within $5 \mathrm{~min}$, the solution ignites fires with flame and finally left with voluminous foamy product (ash). The final foamy product was collected and ground using agate make pestle and mortar.

As-synthesized samples were characterized for phase purity using X-Ray Diffractometer (D8 ADVANCE, Bruker) with wavelength $1.5418 \AA$ and magnetic studies at RT through SQUID magnetometer. Electronic structure and element specific magnetic properties were investigated through NEXAFS and XMCD measurements. X-ray absorption spectroscopy (XAS) i.e., NEXAFS measurements were carried out at different beamlines (BL-11A, 17C and 20A) available at National Synchrotron Radiation Research Center (NSRRC) in Taiwan. Together with SQUID, we also measured the XMCD at 11A1 (Dragon) beamline of NSRRC, Taiwan. All the beamline X-ray absorption data was obtained in the fluorescence yield (FLY) mode, which is mostly bulk sensitive.

\section{Results and discussions}

To synthesize Co-ZnO nanoparticles, for the first time L-Valine has been utilized as a fuel by SCT. Fig. 1 shows the XRD patterns of pure and Co doped $\mathrm{ZnO}$ nanomaterials. From the figure, it is seen that, all peaks could be indexed to the JCPDS card no. 36-1451, belongs to hexagonal wurtzite $\mathrm{ZnO}$ phase with space group P63mc. It is evidenced from the XRD pattern that as synthesized pure and Co substituted $\mathrm{ZnO}$ is in pure single phase, polycrystalline in nature and there is no secondary/impurity phase like, $\mathrm{CoO}_{x}$, metallic Co, etc. It indicates that, 'Co' ions are substituting 'Zn' ions without any

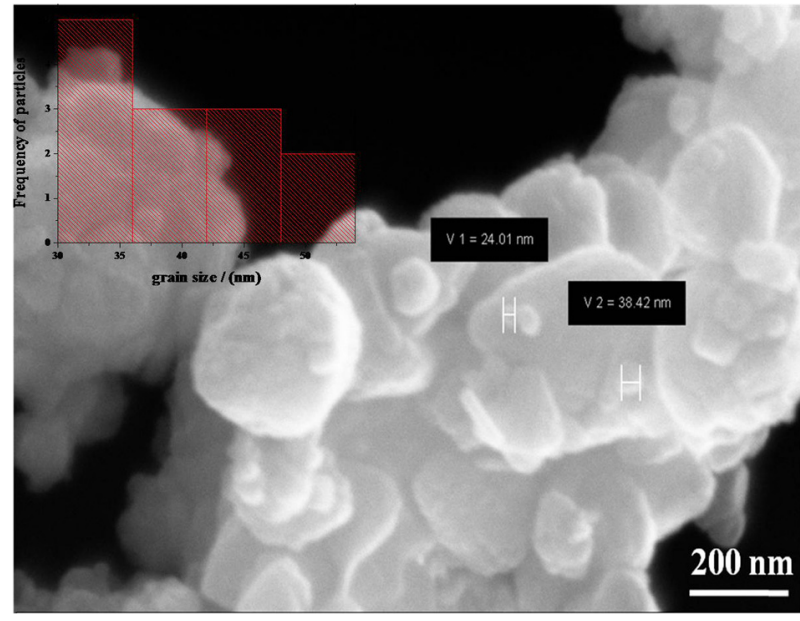

Fig. 2. SEM micrographs of pure $\mathrm{ZnO}$ nano powders. Inset shows the distribution of particles (grains).

secondary phase formation. A shift in the XRD peaks is observed in Fig. 1, which could be due to the increased substitution of 'Co' cation into ' $\mathrm{Zn}$ ' site, which leads to change in the lattice parameters or unit cell volume. The Rietveld refinement was carried out on the XRD data using Fullproof suite. The refined lattice parameters are tabulated in Table 1. The lattice parameters show small variation with increase of ' $\mathrm{Co}$ ' doping in $\mathrm{ZnO}$. This could be due to a very small doping concentration (diluted) $(<5 \%)$ and also could be due to the presence and variation of oxygen vacancies with increase of 'Co' doping. Hence, the variations in lattice parameters are not that significant. These results are in agreement with those reported in literature [24,25].

The crystallite size and strain were calculated using WilliamsonHall Eq. (1).

$\beta \cos \theta=\frac{k \lambda}{D}+4 \varepsilon \sin \theta$

where, $\beta$ is the observed FWHM, $\theta$ is the Bragg angle, $k$ is the Scherer's constant, $\lambda$ is the wavelength of the X-ray used, $D$ is the crystallite size, $\varepsilon$ is the strain present in the crystal.

Crystallite size and strain were determined from the intercept and slope obtained by plotting $\beta \cos \theta$ along $y$-axis and $4 \sin \theta$ along $x$-axis. The obtained values of crystallite size and strain are tabulated in Table 1. It is seen from the table, the average crystallite size found in the range $20-30 \mathrm{~nm}$. The decrease of crystallite size and the strain as a function of ' $\mathrm{Co}$ ' concentration were observed and are attributed to the relative difference in the ionic radii of cations (' $\mathrm{Co}$ ' and ' $\mathrm{Zn}$ '). The substitution of ' $\mathrm{Co}$ ' into ' $\mathrm{Zn}$ ' site would have altered the geometrical structure of the $\mathrm{ZnO}$, thereby introducing lattice micro strain in the material. The crystallite size of the combustion synthesized material is strongly dependent on the exothermicity of the combustion reaction. In this case the inclusion of Co precursor along with the $\mathrm{Zn}$ precursor would have altered the exothermicity of the reaction there by changing the crystallite size.

The micro-structural and surface morphology were studied using scanning electron microscopy. The SEM micrograph of pure $\mathrm{ZnO}$ is depicted in Fig. 2. It is seen from the micrograph that, as synthesized samples are agglomerated, porous in nature and particles are uniformly distributed. It is the indicative sign of evolution of large amounts of nitrate gasses during combustion. Though, as synthesized product (powder) is agglomerated, the average particle (grain) size of $\mathrm{ZnO}$ particles is found to be in the range of nano regime i.e. $30-50 \mathrm{~nm}$ (inset of Fig. 2), it is partial in agreement with the values found from XRD analysis. The particle size distribution is also presented in the form of histogram as an inset in Fig. 2. 
Table 1

Crystallite size, strain, Lattice parameters, coercive field, remnant and saturation magnetizations.

\begin{tabular}{|c|c|c|c|c|c|c|c|c|}
\hline \multirow[t]{2}{*}{ Sample } & \multicolumn{2}{|c|}{ Crystallite size $(D) /(\mathrm{nm})$} & \multirow[t]{2}{*}{ Strain $(\varepsilon) /(\%)$} & \multicolumn{2}{|c|}{ Lattice parameters } & \multirow[t]{2}{*}{$M_{\mathrm{r}} / \times 10^{-5}(\mathrm{emu} / \mathrm{g})$} & \multirow[t]{2}{*}{$H_{\mathrm{c}} /(\mathrm{Oe})$} & \multirow[t]{2}{*}{$M_{\mathrm{s}} / \times 10^{-5}(\mathrm{emu} / \mathrm{g})$} \\
\hline & XRD & TEM & & $a=b /(\AA)$ & $c /(\AA)$ & & & \\
\hline Pure ZnO & 30.58 & 33.0 & 0.256 & $3.251(1)$ & $5.206(1)$ & - & - & - \\
\hline $\mathrm{Zn}_{0.99} \mathrm{Co}_{0.01} \mathrm{O}$ & 28.70 & 31.0 & 0.231 & $3.252(1)$ & $5.206(1)$ & 11.2 & 176 & 35.7 \\
\hline $\mathrm{Zn}_{0.97} \mathrm{Co}_{0.03} \mathrm{O}$ & 22.34 & - & 0.183 & $3.254(1)$ & $5.209(1)$ & 28.1 & 312 & 56.5 \\
\hline $\mathrm{Zn}_{0.95} \mathrm{Co}_{0.05} \mathrm{O}$ & 20.45 & - & 0.161 & $3.253(1)$ & $5.207(1)$ & 33.0 & 378 & 131.4 \\
\hline
\end{tabular}
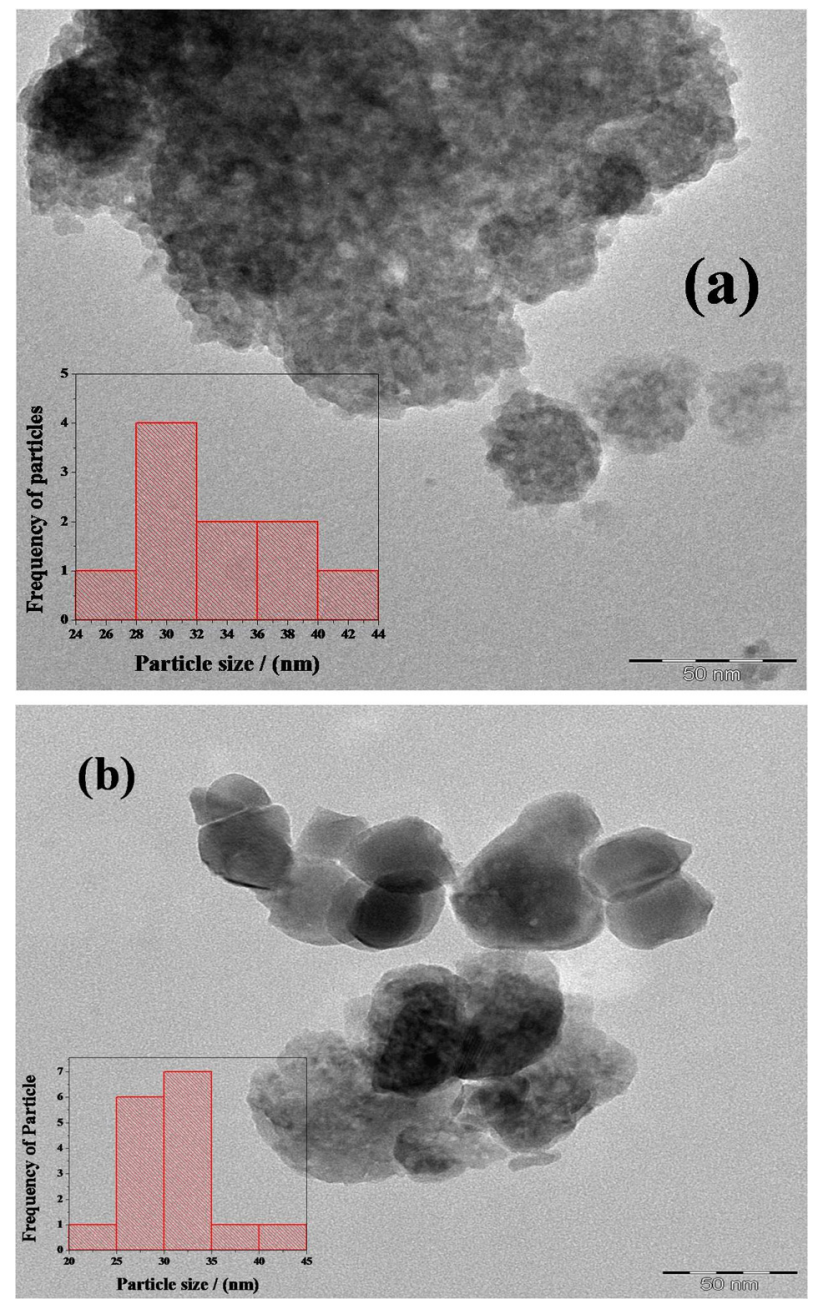

Fig. 3. TEM micrographs of (a) Pure $\mathrm{ZnO}$, and (b) $\mathrm{Zn}_{0.99} \mathrm{Co}_{0.01} \mathrm{O}$. Insets show their corresponding distributions of the particles.

The transmission electron microscopy (TEM) micrographs of pure $\mathrm{ZnO}$ and $\mathrm{Zn}_{0.99} \mathrm{Co}_{0.01} \mathrm{O}$ are depicted in Fig. 3. It is found from Fig. 3 that, the particles are agglomerated, having wide distribution ranging from 15 to $50 \mathrm{~nm}$ for pure $\mathrm{ZnO}$ and 30 to $45 \mathrm{~nm}$ for $\mathrm{Zn}_{0.99} \mathrm{Co}_{0.01} \mathrm{O}$. The average particle sizes estimated from the TEM micrographs are found to be $33 \mathrm{~nm}$ and $31 \mathrm{~nm}$ for pure $\mathrm{ZnO}$ and $\mathrm{Zn}_{0.99} \mathrm{Co}_{0.01} \mathrm{O}$, respectively. These values are in agreement with those obtained from XRD and SEM measurements.

The RT $(300 \mathrm{~K})$ magnetization with applied magnetic field (M-H loop) measurements were carried out for as synthesized $\mathrm{Zn}_{1-x} \mathrm{Co}_{x} \mathrm{O}$ $(x=0,0.01,0.03,0.05)$ powders through SQUID magnetometer. The obtained magnetic-hysteresis (M-H) loops are depicted in Fig. 4. It is seen from the RT M-H loops that the $x=0$ (top-left inset of Fig. 4) exhibits diamagnetic behavior with the negative slope of the curve, which is due to the diamagnetic nature of pure $\mathrm{ZnO}$. After removing the contribution (straight line) of the paramagnetic

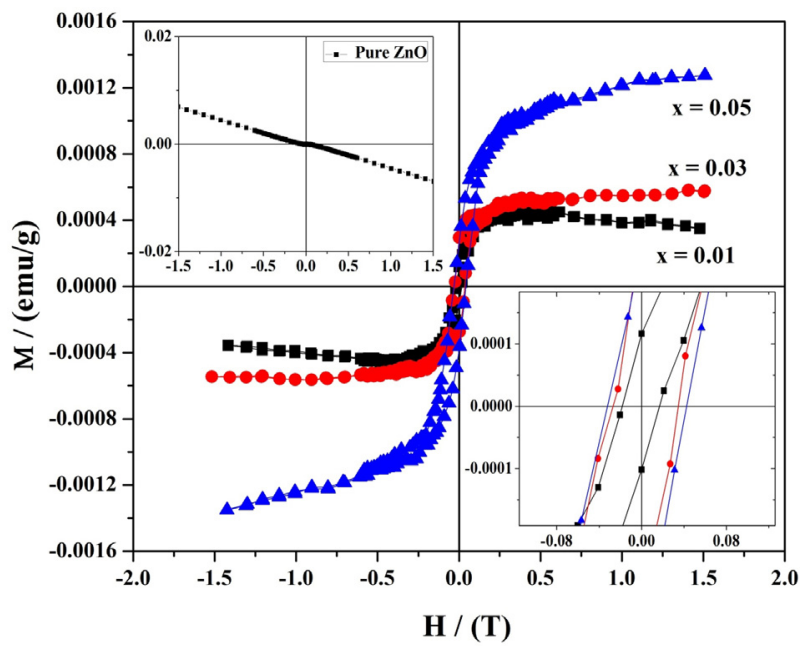

Fig. 4. Magnetic hysteresis plots at $300 \mathrm{~K}$ for $\mathrm{Zn}_{1-x} \mathrm{Co}_{x} \mathrm{O}$. Top-left inset shows the diamagnetic behavior for $x=0.00$ and bottom-right inset shows enlarged part hysteresis loops.

phase of other samples $\mathrm{Zn}_{1-x} \mathrm{Co}_{x} \mathrm{O}(x=0.01,0.03,0.05)$, exhibit ferromagnetic behavior with clear hysteresis loops (down-right inset of Fig. 4) at room temperature due to the incorporation of 'Co' ions into the $\mathrm{ZnO}$ host lattice. The remnant magnetization $\left(M_{\mathrm{r}}\right)$, Coercive field and their corresponding saturation magnetization $\left(M_{\mathrm{S}}\right)$ values for $\mathrm{Zn}_{1-x} \mathrm{Co}_{x} \mathrm{O}(x=0.01,0.03,0.05)$ increases with 'Co' concentration and are tabulated below in Table 1 . The existence of RTFM is attributed to the incorporation of small amount of ' $\mathrm{Co}$ ' ions into the $\mathrm{ZnO}$ host lattice. Though no impurities were observed within in the detection limit of XRD, there can be possible amount of impurities below the XRD detection limit. Hence for further confirmation of intrinsic ferromagnetism in $\mathrm{Co}-\mathrm{ZnO}$, the NEXAFS and XMCD studies are carried out.

To further investigate the role of 'Co' doping and to ascertain of its position in ZnO host lattice, NEXAFS and XMCD measurements on $\mathrm{Co}-\mathrm{ZnO}$ nanoparticles have been carried out. NEXAFS and XMCD are considered to be powerful tools to investigate the electronic structure and magnetic properties of Co doped $\mathrm{ZnO}$ nanoparticles. The line shape of NEXAFS spectra is the fingerprint for $\mathrm{d}$ - as well as p-state configuration. The NEXAFS is an element-specific technique, in which the spectral features are very much sensitive to the local environment of the probed ion. This tool can be used to determine the charge state of 'Co' ion in the $\mathrm{ZnO}$ host and to determine the presence of ' $\mathrm{Co}$ ' ion as clusters or in any other oxide phase or whether it is substituting at the 'Zn' site in the host ZnO. Also, XMCD is one of the tools to investigate the source of magnetism, the role of 'Co' ions in the $\mathrm{ZnO}$ host lattice and to determine the magnetic contribution from the spin and orbital part using sum rules [22,23]. NEXAFS spectra were collected at O K-, Zn $L$ - and Co $L$ - edges and XMCD spectra were taken for fixed helicity of the light.

Incorporation of ' $\mathrm{Co}$ ' ions into the $\mathrm{ZnO}$ host lattice would have altered $\mathrm{O} K$-edge NEXAFS spectra of ZnO. Fig. 5 shows the normalized O K-edge NEXAFS spectra at various 'Co' concentrations, which represents the orbital nature of the spectral features of the 


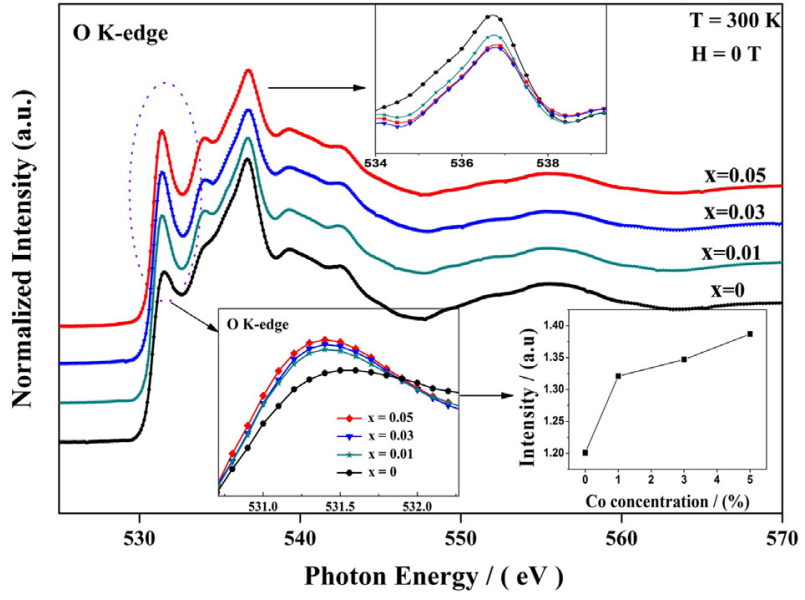

Fig. 5. NEXAFS spectra of $\mathrm{Zn}_{1-x} \mathrm{Co}_{x} \mathrm{O}$ nanoparticles taken at $\mathrm{O} \mathrm{K}$-edge.

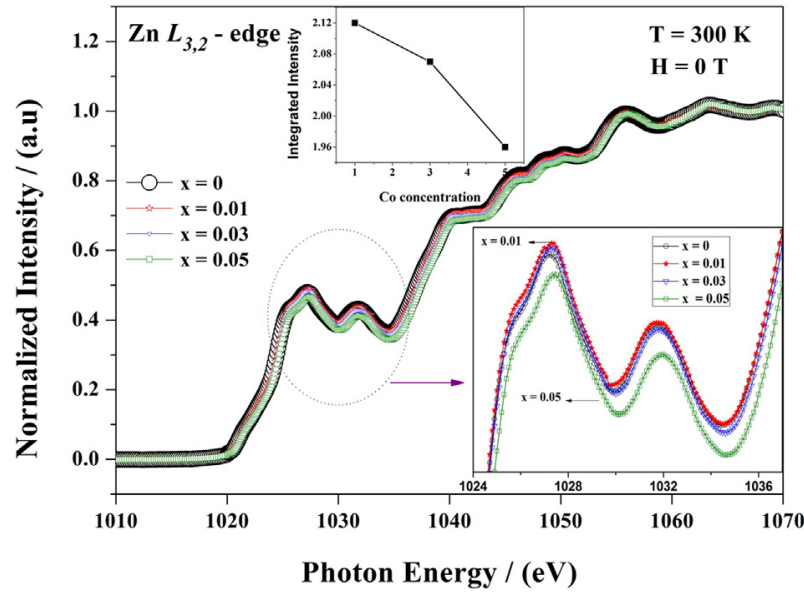

Fig. 6. NEXAFS spectra $\mathrm{Zn}_{1-x} \mathrm{Co}_{x} \mathrm{O}$ nanoparticles taken at $\mathrm{Zn} L_{3,2}$-edge.
O $2 p$ unoccupied states in the conduction band and its hybridization with different ' $Z n$ ' and 'Co' orbitals. It is seen that, the spectral features of both pure and Co doped $\mathrm{ZnO}$ exhibits several prominent features. Based on the literature [26,27], the spectral features are assigned as follows. In the case of both pure and Co doped $\mathrm{ZnO}$ : (i) the features in the energy region between 532 and $539 \mathrm{eV}$ are assigned to the hybridization of $\mathrm{O} 2 p$ with highly dispersive $\mathrm{Zn}$ $3 d 4 s /$ Co $3 d$ states, forming the bottom of the conduction band with prominent peak at $536.7 \mathrm{eV}$ due to the transition of $\mathrm{O} 1 \mathrm{~s}$ electrons to more localized (non-dispersive) $02 p$ states $\left(2 p_{z}\right.$ and $2 p_{x+y}$ ) [28], (ii) the features between 539 and $547 \mathrm{eV}$ are assigned for the transition to the states formed due to the hybridization between $\mathrm{O} 2 p$ states with $\mathrm{Zn} 4 p$ and Co $4 p$ states and (iii) the spectral features above $547 \mathrm{eV}$ is emerged due to the hybridization of $\mathrm{O} 2 p$ states with the extended higher orbitals of 'Zn' and 'Co' [29]. As 'Co' concentration increases in $\mathrm{ZnO}$, there is an increase in the intensity of the pre-edge peak at $531.6 \mathrm{eV}$ (see bottom-inset figure). This is due to the fact that, as 'Co' concentration increases, more and more 'Co' unoccupied $3 d$ states are being introduced within the band gap of the $\mathrm{ZnO}$ that hybridizes with the $\mathrm{O} 2 p$ orbitals. The appearance of similar feature has been reported [30-32] in case of thin films. It is also evidenced that, this pre-edge peak also present in the case of pure $\mathrm{ZnO}$. If the enhancement in pre-edge peak at $531.6 \mathrm{eV}$ is attributed to the incorporation of ' $\mathrm{Co}$ ' ions (concentrations), no peak is expected in the case of pure $\mathrm{ZnO}$, since ' $\mathrm{Zn}$ ' has $3 d^{10}$ configuration; as no empty $3 d$ states are available. However, the presence of this pre-edge peak is attributed due to some ' $O$ ' vacancies or some other defects present in the sample, which might be introduced during the combustion synthesis. Apart from these features, other noticeable changes in the spectral features were observed. The intensity of peak at $536.7 \mathrm{eV}$ decreases with increase in 'Co' concentration (see top-inset figure) and the broadening of other peaks with energy greater than around $538 \mathrm{eV}$. Similar features and broadening were reported in [28,31]. These broadenings are attributed to the presence of ' $\mathrm{O}$ ' vacancies and 'Co' doping. Due to the hybridization of $\mathrm{Co} 3 d$ states with $\mathrm{O} 2 p$ states, the $\mathrm{O} 2 p$ states becomes more and more dispersive, leading to the broadening of the spectral features with increase in the 'Co' concentration.

According to the dipole transition selection rule, $\mathrm{Zn} L_{3,2}$-edge NEXAFS probes the unoccupied $\mathrm{Zn} s$ - and $p$ - derived states. Fig. 6 represents $\mathrm{Zn} L_{3,2}$-edge NEXAFS spectra of pure and Co doped $\mathrm{ZnO}$ nanoparticles. The spectral features in the energy region between 1020 and $1035 \mathrm{eV}$ are associated with the transition of $\mathrm{Zn} 2 p$ electron to $\mathrm{Zn} 4 s$ and anti-bonding $3 d$ states [33]. The bottom inset reveals the intensities of features around $1028 \mathrm{eV}$ and $1032 \mathrm{eV}$ that decreases and shifts toward higher energies with increase of ' $\mathrm{Co}$ ' concentration, where as the features around $1022 \mathrm{eV}$ are less sensitive to the 'Co' doping. This is because, $3 d$ orbitals are more localized than the $4 s$ orbitals, the transition probability of $\mathrm{Zn} 2 p$ to $3 d$ would be larger than that of $\mathrm{Zn} 2 p$ to $4 s$. Hence these features will be dominated by $\mathrm{Zn} 3 d$ states contributions. The decrease in the intensities (integrated intensities) of the above features (top inset) indicate that the number of unoccupied $\mathrm{Zn} 4 s$ and $4 d$ states near the conduction band minimum are reduced due to the incorporation of ' $\mathrm{Co}$ ' ions.

The NEXAFS at Co $L_{3,2}$-edge determines the $3 d$ occupancy of the ' $\mathrm{Co}$ ' ions and hence provides an information on the charge state of the 'Co' ions in the ZnO host lattice. The NEXAFS spectra of Co doped $\mathrm{ZnO}$ taken at $\mathrm{Co} L_{3,2}$-edge is depicted in Fig. 7. It is seen from the spectra, the features for all 'Co' concentrations are same except the variation in the intensities. The spectral features between 775 and $785 \mathrm{eV}$ exhibit multiple absorption peaks, which are assigned to the Co $2 p_{3 / 2}-3 d_{5 / 2}$ ( $L_{3}$-edge) transitions, and those in the region $790-798 \mathrm{eV}$ to the Co $2 p_{1 / 2}-3 d_{3 / 2}$ ( $L_{2}$-edge) transitions. The large energy separation between these absorption lines is due to spinorbit splitting of the $2 p$ core holes. The intensity of spectral features of $L_{3}$-edge increases with increase of 'Co' concentration (inset of Fig. 7), due to the more and more Co $3 d$ unoccupied states are being introduced into the $\mathrm{ZnO}$ band gap region; hence increase in the transition probability. The observed spectral features were

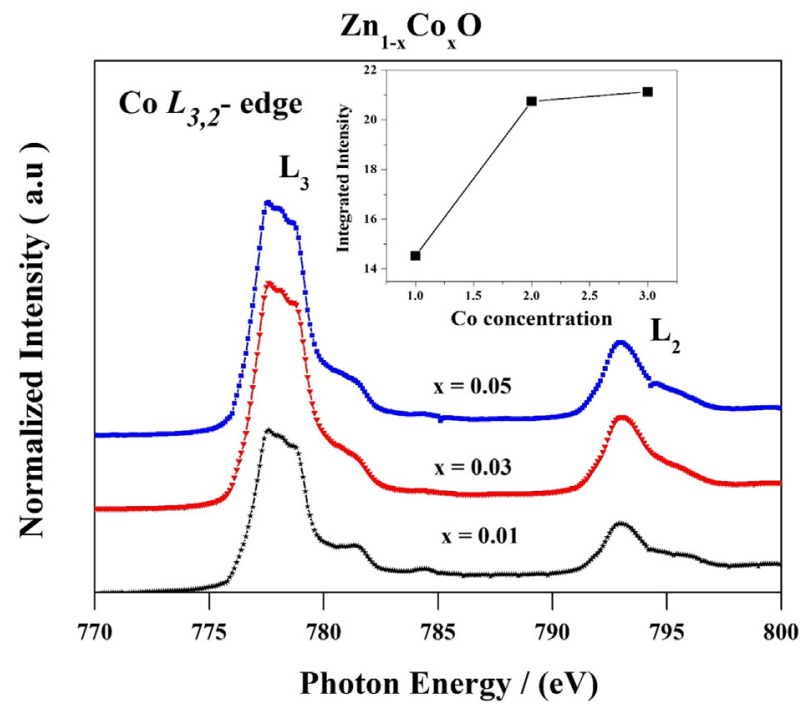

Fig. 7. NEXAFS spectra of $\mathrm{Zn}_{1-x} \mathrm{Co}_{x} \mathrm{O}$ nanoparticles taken at $\mathrm{Co}_{3,2}$-edge. 

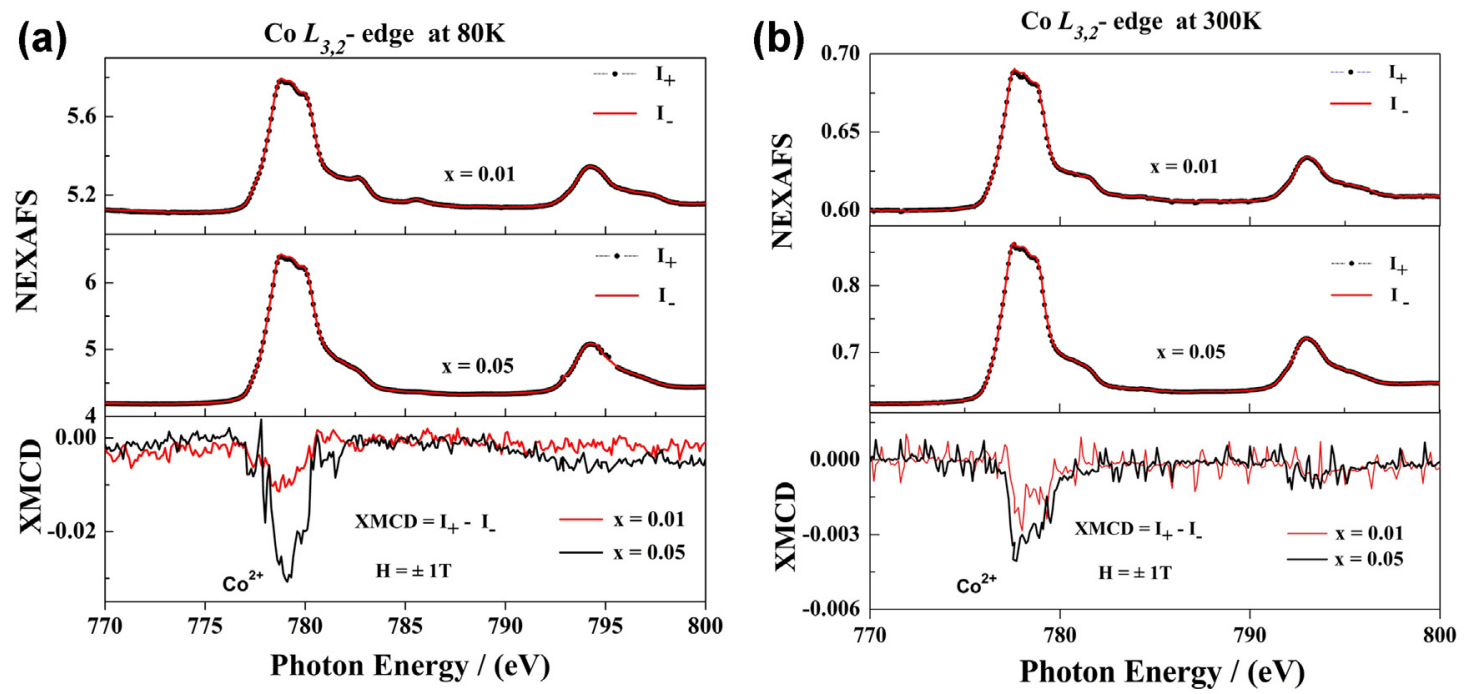

Fig. 8. XMCD spectra of $\mathrm{Zn}_{1-x} \mathrm{Co}_{x} \mathrm{O}$ nanoparticles taken at $\mathrm{Co} L_{3,2}$-edge with $H= \pm 1 \mathrm{~T}$.

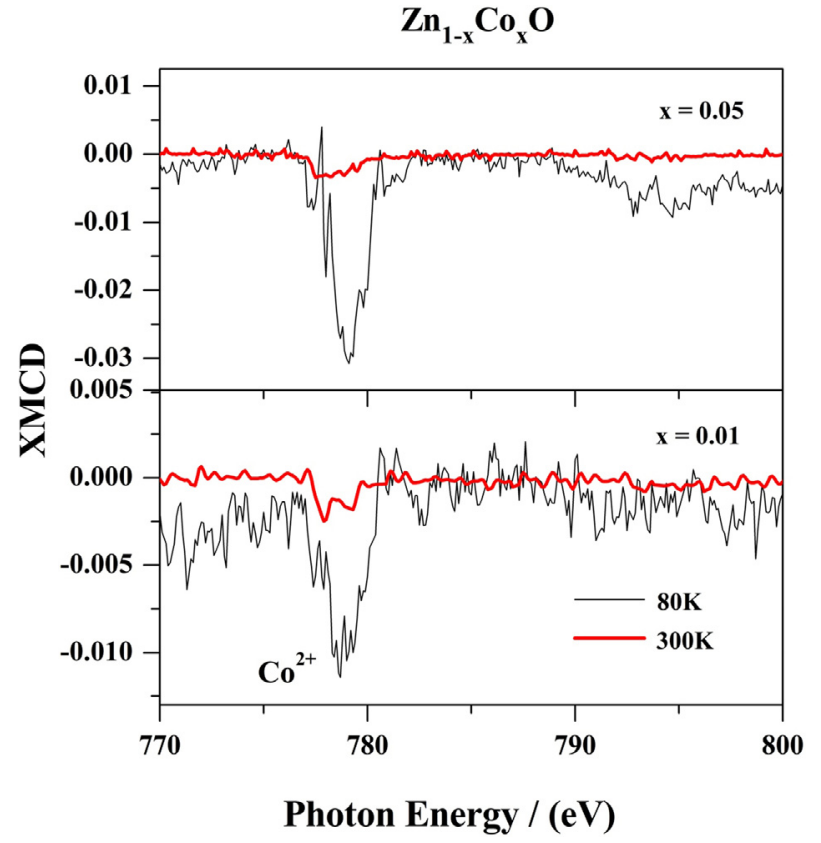

Fig. 9. Temperature dependent XMCD spectra of $\mathrm{Zn}_{1-x} \mathrm{Co}_{x} \mathrm{O}$ nanoparticles taken at Co $L_{3,2}$-edge with $H= \pm 1 \mathrm{~T}$.

compared with the existing theoretical spectral features based on the atomic multiplet calculations found from the literature [31,32], it is concluded that, the valence state of 'Co' is found to be +2 , it is in tetrahedral symmetry surrounded by four ' $O$ ' atoms with crystalfield splitting value of $10 D q=-0.6 \mathrm{eV}$. Also no 'Co' clusters/impurity phases, $\mathrm{Co}_{3} \mathrm{O}_{4}$ is present in the sample. Hence, 'Co' is substituted at the ' $\mathrm{Zn}$ ' site in the $\mathrm{ZnO}$ host lattice.

To further investigate the source of magnetism and the role of ' $C o$ ' in the ZnO host lattice, the XMCD spectra were collected at the Co $L_{3,2}$-edge at $80 \mathrm{~K}$ and $300 \mathrm{~K}$. XMCD spectra is depicted in Fig. 8. The XMCD signal is the difference between the NEXAFS spectra recorded for the parallel $\left(\mathrm{I}^{+}\right)$and anti-parallel $\left(\mathrm{I}^{-}\right)$alignments of the photon helicity with the applied magnetic field $( \pm 1 \mathrm{~T})$. It is seen from the XMCD $\left(\mathrm{I}^{+}\right.$to $\left.\mathrm{I}^{-}\right)$signal with a negative sign at $h \nu=\sim 777 \mathrm{eV}$ in case of RT, and $\sim 779 \mathrm{eV}$ for $80 \mathrm{~K}$ confirms the magnetic contribution of $\mathrm{Co}^{2+}$ ions, which are responsible for the FM ordering at RT and at $80 \mathrm{~K}$, respectively. The relative intensity of XMCD signal

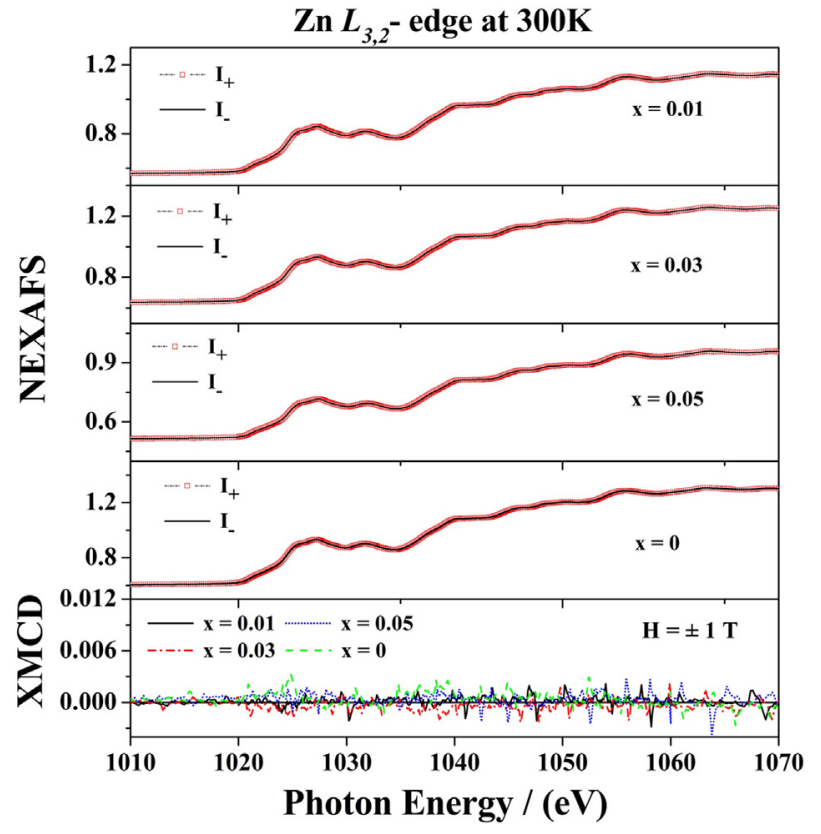

Fig. 10. Normalized NEXAFS and XMCD spectra of pure $\mathrm{ZnO}$ and $\mathrm{Zn}_{1-x} \mathrm{Co}_{x} \mathrm{O}(x=0.01$, 0.03 and 0.05 ) taken at $\mathrm{Zn} L_{3,2}$-edge with $H= \pm 1 \mathrm{~T}$.

at $L_{3}$ edge increases with 'Co' concentrations, due to the increase of 'Co' ions in the host lattice. It is also seen that, there is a weak signal at the $L_{2}$-edge; this would suggests the presence of large orbital contribution of the magnetic moment $[30,32,34]$.

The intensities of XMCD signals decrease with temperature, which could be due to the paramagnetic contribution from 'Co' ions. Further to confirm this, the temperature-dependent XMCD spectra are depicted in Fig. 9. There is a considerable difference between the XMCD signal collected at $80 \mathrm{~K}$ and $300 \mathrm{~K}$. This difference in the intensities of XMCD signal collected at $80 \mathrm{~K}$ and $300 \mathrm{~K}$ indicates that there is a considerable contribution of the ' $\mathrm{Co}$ ' ions toward the magnetism of the system, which is in consistence with the result obtained by [35]. They observed a decrease in XMCD signal at the Co $L_{3,2}$-edge with increase in temperature, in our case almost similar trend has been observed. Thus we conclude that, the contribution of doped 'Co' ions toward the magnetism in the system is FM with considerable paramagnetic component. The sum rules can be used 
to determine the orbital and spin contribution to the total magnetization, but the values obtained would be unreliable, since there is no single emerging at $L_{2}$-edge.

In order to further investigate the origin of FM in our $\mathrm{Zn}_{1-x} \mathrm{Co}_{x} \mathrm{O}$ $(x=0,0.01,0.03$ and 0.05$)$, we searched for the possible magnetic response of the ' $\mathrm{Zn}$ ' sub-lattice. The electronic configuration for $\mathrm{Zn}^{2+}$ is formally $3 d^{10}$, so that a $3 d$ magnetic moment cannot be expected to be present. However, the presence of vacancies and/or interstitial ' $\mathrm{Zn}$ ' atoms could be at the origin of an unfilled $3 d$ shell, which could, in turn, carry a magnetic moment. The above Fig. 10 shows the NEXAFS and XMCD spectra measured at the $Z n L_{3,2}$-edges at $H= \pm 1 \mathrm{~T}$. The NEXAFS spectra did not show the presence of any strong white line, as expected for a fairly pure $3 d^{10}$ configuration. And also, there is no evidence of an XMCD signal in the measured energy region. This rules out the presence of any detectable ferromagnetic contribution of the $\mathrm{Zn} s$ and $d$ shells.

\section{Conclusions}

We have successfully synthesized single-phase Co:ZnO diluted magnetic semiconductor nanoparticles using combustion technique with L-Valine as a fuel for the first time. The XRD pattern reveals the formation of single-phase and an absence of secondary phases of 'Co' metal or 'Co' oxides. Formation of nanoparticles was confirmed through SEM micrographs in agreement with the values obtained from XRD. SQUID measurements reveals as synthesized Co doped $\mathrm{ZnO}$ nanoparticles exhibits carrier mediated RTFM which is intrinsic due to the incorporation of ' $\mathrm{Co}$ ' ions. Hence XRD and SQUID measurements confirm the substitution of 'Co' at ' $\mathrm{Zn}$ ' site in the $\mathrm{ZnO}$ host lattice. The effect of Co substitution on the spectral features of $\mathrm{Co}-\mathrm{ZnO}$ at $\mathrm{O}$-edge, Co $L_{3,2}$ edge, $\mathrm{Zn} L_{3,2}$ edge have been investigated. The $\mathrm{O} K$-edge NEXAFS spectra reveals the strong hybridization of $\mathrm{O} 2 p$ states with Co $3 d$ orbitals, which is evident from the increase in the intensity of pre-edge spectral feature as a function of 'Co' concentration. From the Co $L$-edge NEXAFS spectra the ionic state of 'Co' ions in $\mathrm{ZnO}$ host was found to be in +2 state. XMCD spectra at RT show that the magnetic contribution is due to $\mathrm{Co}^{2+}$ ions, not due to the Co metal cluster. Hence, $\mathrm{Zn}_{1-x} \mathrm{Co}_{x} \mathrm{O}$ nanoparticles exhibit intrinsic ferromagnetism at room temperature with considerable weak paramagnetic contribution as revealed by the temperature-dependent XMCD studies. This paramagnetic contribution was coming due to the presence of ' $O$ ' vacancies or 'Co' incorporation, which is consistent with $\mathrm{O} K$-edge results. Over all the spectroscopic results reveal that the ferromagnetic behavior is intrinsic, this is prerequisite for spintronic applications.

\section{Acknowledgments}

Authors (S.N and B.A) are grateful to UGC-DAE-CSR, Kalpakkam Node for the financial support through CRS No. CSR-KN/CRS-22. NGD and WFP are thankful to National Synchrotron Radiation Research Center (NSRRC), Taiwan for providing beamline to carry out NEXAFS and XMCD measurements. Authors thank Dr. S. Amrithapandian, UGC-DAE-CSR/IGCAR, Kalpakkam for TEM measurements.

\section{References}

[1] H. Ohno, Science 281 (1998) 951.

[2] Y. Ohno, D.K. Young, B. Beschoten, F. Matsukura, H. Ohno, D.D. Awschalom, Nature 402 (1999) 790-792.

[3] S.J. Pearson, W.H. Heo, M. Ivill, D.P. Norton, T. Steiner, Semicond. Sci. Technol. 19 (2004) R59-R74.

[4] T. Dietl, H. Ohno, F. Matsukura, J. Cibert, D. Ferrand, Science 287 (2000) 1019.

[5] K. Sato, H. Katayama-Yoshida, Jpn. J. Appl. Phys. 39 (2000) L555.

[6] P.K. Sharma, R.K. Dutta, A.C. Pandey, S. Layek, H.C. Verma, J. Magn. Magn. Mater. 321 (2009) 2587-2591.

[7] M.L. Dinesha, H.S. Jayanna, S. Mohanty, S. Ravi, J. Alloys Compd. 490 (2010) $618-623$.

[8] R.K. Singhal, A. Samariya, Y.T. Xing, S. Kumar, S.N. Dolia, U.P. Deshpande, T. Shripathi, B.E. Saitovitch, J. Alloys Compd. 496 (2010) 324-330.

[9] F. Ahmed, S. Kumar, N. Arshi, M.S. Anwar, B.H. Koo, C.G. Lee, Microelectron. Eng. 89 (2012) 129-132.

[10] J. Mohapatra, D.K. Mishra, S.K. Singh, A. Mahato, A. Mitra, Nanosci. Nanotechnol. Lett. 5 (2013) 563-567.

[11] B. Angadi, Y.S. Jung, W.K. Choi, R. Kumar, K. Jeong, S.W. Shin, J.H. Lee, J.H. Song M. Wasi Khan, J.P. Srivastava, Appl. Phys. Lett. 88 (2006) 142502.

[12] R. Kumar, F. Singh, B. Angadi, J.W. Choi, W.K. Choi, K. Jeong, J.H. Song, M. Wasi Khan, J.P. Srivastava, R.P. Tandon, J. Appl. Phys. 100 (2006) 113708.

[13] R. Kumar, A.P. Singh, P. Thakur, K.H. Che, W.K. Choi, B. Angadi, S.D. Kaushik, S. Patnaik, J. Phys. D 41 (2008) 155002.

[14] A. Ney, V. Ney, S. Ye, K. Ollefs, T. Kammermeier, T.C. Kaspar, S.A. Chambers, F Wilhelm, A. Rogalev, Phys. Rev. B 82 (2010) 041202(R).

[15] C.C. Wang, M. Liu1, B.Y. Man, C.S. Chen, S.Z. Jiang, S.Y. Yang, X.G. Gao, S.C. Xu, B. Hu, Z.C. Sun, J.J. Guo, J. Hou, AIP Adv. 2 (2012) 012182.

[16] K.A. Aravind, K. Hasna, M.K. Jayaraj, M. Kumar, R. Chandra, Appl. Phys. A (2013), http://dx.doi.org/10.1007/s00339-013-7875-0.

[17] B. Loukya, D.S. Negi, K. Dileep, N. Kumar, J. Ghatak, R. Datta, J. Magn. Magn. Mater. 345 (2013) 159-164.

[18] A. Popa, D. Toloman, O. Raita, A.R. Biris, G. Borodi, T. Mustafa, F. Watanabe, A.S. Biris, A. Darabont, L.M. Giurgiu, Central Eur. J. Phys. 9 (6) (2011) 1446.

[19] Gu Hao, Zhang Wen, Xu Yongbing, Yan. Mi, Appl. Phys. Lett. 100 (2012) 202401

[20] H. Gu, Y. Jiang, Y. Xu, M. Yan, Appl. Phys. Lett. 98 (2011) 012502

[21] H. Gu, Y. Jiang, Y. Xu, M. Yan, Appl. Phys. A 107 (4) (2012) 919

[22] B.T. Thole, P. Carra, F. Sette, G. van der Laan, Phys. Rev. Lett. 68 (1992 1943.

[23] P. Carra, B.T. Thole, M. Altarelli, X. Wang, Phys. Rev. Lett. 70 (1993) 694

[24] A.C. Tuan, J.D. Bryan, A.B. Pakhomov, V. Shutthanandan, S. Thevuthasan, D.E. McCready, D. Gaspar, M.H. Engelhard, J.W. Rogers Jr., K. Krishnan, D.R. Gamein, S.A. Chambers, Phys. Rev. B 70 (2004) 054424.

[25] Y.Q. Chang, P.W. Wang, S.L. Ni, Y. Long, X.D. Li, J. Mater. Sci. Technol. 28 (4) (2012) 313.

[26] J.-H. Guo, L. Vayssieres, C. Persson, R. Ahuja, B. Johansson, J. Nordgren, J. Phys.: Condens. Matter 14 (2002) 6969.

[27] C.L. Dong, C. Persson, L. Vayssieres, A. Augustsson, T. Schmitt, M. Mattesini, R Ahuja, C.L. Chang, J.-H. Guo, Phys. Rev. B 70 (2004) 195325.

[28] S. Krishnamurthy, C. McGuinness, L.S. Dorneles, M. Venkatesan, J.M.D. Coey, J.G. Lunney, C.H. Patterson, K.E. Smith, T. Learmonth, P.-A. Glans, T. Schmitt, J.-H. Guo, J. Appl. Phys. 99 (2006) 08M111.

[29] T. Tietze, M. Gacic, G. Schtz, G. Jakob, S. Brck, E. Goering, New J. Phys. 10 (2008) 055009.

[30] K.H. Thakur, J.-Y. Chae, M. Kim, R. Subramanian, K. Jayavel, Asokan, Appl. Phys. Lett. 91 (2007) 162503.

[31] A.P. Singh, R. Kumar, P. Thakur, N.B. Brookes, K.H. Chae, W.K. Choi, J. Phys: Condens. Matter 21 (2009) 185005

[32] S. Goutam, P. Thakur, P. Bazylewski, R. Bauer, A.P. Singh, J.Y. Kim, M. Subramanian, R. Jayavel, K. Asokan, K.H. Chae, G.S. Chang, Mater. Chem. Phys. 140 (2013) $130-134$.

[33] J.W. Chiou, J.C. Jan, H.M. Tsai, C.W. Bao, W.F. Pong, M.-H. Tsai, I.-H. Hong, R. Klauser, J.F. Lee, J.J. Wu, S.C. Liu, Appl. Phys. Lett. Vol. 85 (15) (2004) 3462-3464.

[34] T. Frunk, A. Deb, S.J. George, H. Wang, S.P. Cramer, Coord. Chem. Rev. 249 (2005) 3-30.

[35] A. Barla, G. Schmerber, E. Beaurepaire, A. Dinia, H. Bieber, S. Colis, F. Scheurer, J.-P. Kappler, P. Imperia, F. Nolting, F. Wilhelm, A. Rogalev, D. Müller, J.J. Grob, Phys. Rev. B 76 (2007) 125201. 